\title{
Recent mathematical-computational techniques and models in chemistry
}

\author{
Jesús Vigo-Aguiar ${ }^{1} \cdot$ Higinio Ramos $^{1}$
}

Guest Editors Foreword for the Special Collection of Papers Devoted to "Recent computational techniques and models in chemistry".

The sixteenth international conference "Computational and Mathematical Methods in Science and Engineering" (CMMSE 2016) was held in Rota (Cádiz, Spain), July 4-8, 2016. More than 250 participants from all around the world were attending the conference. This international conference was organized by the University of Cádiz, Spain, and was chaired by Jesús Vigo-Aguiar, Department of Applied Mathematics, University of Salamanca, Spain, and co-chaired by Ian P. Hamilton, Wilfrid Laurier University, Canada, and by Bruce A. Wade, University of Wisconsin, USA.

The purpose of this meeting was to serve as a catalyst for new advances in computational mathematics and for cross-fertilization among cutting edge applications in science and engineering, especially in areas connected to chemistry, and to provide a forum exchanging new ideas and discussing future developments. The large number of papers presented at the conference addressing topics related to mathematical chemistry illustrates the close relationship between chemistry and mathematics.

This issue of the Journal of Mathematical Chemistry contains carefully selected and refereed papers presented by participants of the conference.

We are happy to have been able to collect such manuscripts at the leading edge of research on mathematical aspects of chemistry from nine of the presenters at the conference. We hope that the readers of this issue will enjoy these contributions as much as we have.

Jesús Vigo-Aguiar

jvigo@usal.es

Higinio Ramos

higra@usal.es

1 Universidad de Salamanca, Salamanca, Spain 
In the article of Calvo and Yurtsever, the solvation of Buckminsterfullerene $\mathrm{C}_{60}$ by para-hydrogen and orthodeuterium clusters are discussed, considering a model through a dedicated potential and path-integral molecular dynamics simulations at low temperature $(2 \mathrm{~K})$. The solvation shell obtained from the distribution of radial distances is found to involve nearly 50 molecules, in agreement with recent mass spectrometry measurements.

The original concept of geometric-arithmetic index has been introduced in the chemical graph theory some time ago (H. Wiener, 1947), but since then, and even recently, many useful papers studying various types of indices have appeared. The contribution by Hernández, Rodríguez, and Sigarreta shows that the computation of the geometric-arithmetic index of a molecular graph $\mathrm{G}$ may be reduced to the computation of the geometric-arithmetic indices of the so-called primary subgraphs obtained by a general decomposition of $\mathrm{G}$. Using this result additional formulas for the geometricarithmetic indices of various classes of graphs are obtained.

Many problems of chemistry are formulated by means of specific systems of equations. The numerical treatment of the questions related to the solution of such systems requires various branches of computational mathematics as applied for chemistry. Newton's method has a long history in solving nonlinear equations and there is a huge amount of results concerning the convergence of this method under mild differentiability conditions. In the paper by Argyros, Ezquerro, Hernández-Verón, and Magreñán, the convergence domain of Newton's method is extended without imposing additional hypotheses, providing tighter error bounds on the differences between the iterates and the true solution.

In the article "DRBEM solution of biomagnetic fluid flow and heat transfer in cavities", by Senel and Tezer-Sezgin, the investigation is focused on the flow of an electrically non-conducting, viscous, biomagnetic fluid in the $2 \mathrm{D}$ cross section (cavity) of a long impermeable pipe. The study of biomagnetic fluid flow under the effect of a magnetic field has many medical applications. The dual reciprocity boundary element method (DRBEM) with constant and linear elements is used for solving the relevant equations; this is done at a considerably lower computational cost due to the special characteristics of the DRBEM method.

Argyros, Magreñán, Orcos, and Sicilia presented a local convergence analysis for a relaxed two-step Newton-like method considering hypotheses on the first Fréchet derivative and on the central divided-difference of order one. Some numerical examples and an application to Planck's Radiation Law problem calculating the energy density within an isothermal black body are provided to validate the general results.

In the contribution of Cordero, Torregrosa, and Vassileva a new family of parametric iterative methods of arbitrary order is constructed, based on the extension of Ostrowski's and Chun's methods for solving nonlinear systems. The proposed methods may be used for solving the nonlinear system of equations obtained after discretizing the system of partial differential equations that is able to model many problems (such as those related to gas dynamics, heat transfer, filtration of liquids, elasticity, and chemical reactions).

The paper by Cordero, Maimó, Torregrosa, and Vassileva focuses on a multidimensional dynamical approach of the Ostrowsky-Chun family of iterative methods for solving systems of nonlinear equations. This study is important in analyzing the 
stability and reliability of the applied methods. Using their approach, the method can provide a completely stable performance, and also criteria for regions where it is problematic to choose values for any of the parameters. Some actual problems are presented to illustrate the general results.

In the article "Two mathematical models for dissolved oxygen in a lake", Bulai and Venturino introduce new models for handling water pollution. In the first model it is assumed that algae and fungi are in competition for resources that come from wastewater, while in the second model an equation to represent nutrients is explicitly introduced. The qualitative behavior of both models is discussed, determining also the equilibrium points.

In the article of Magreñan, Argyros, and Sicilia, a new semilocal convergence analysis for Newton-like methods is presented, using restricted convergence domains in a Banach space setting. This new approach improves the convergence analysis when compared to the computational cost when the Lipschitz constants are involved. Some numerical studies including a chemical application are presented.

The Guest Editors thank all the authors and referees for their valuable contributions to this special collection. Also, thanks are due to Professor Paul G. Mezey, Editor-inChief, for his invaluable cooperation throughout process. 